\title{
Immunohistochemistry: a rapid and specific diagnostic tool for influenza virus infection in pigs
}

\begin{abstract}
During the 2016 spring, a local outbreak of severe dyspnea, seromucous nasal secretion, anorexia and loss of productive performance was identified in an intensive pig farm in southern Chile. Samples of diseased animals were submitted to the Diagnostic Laboratory of the Faculty of Veterinary Medicine, in Chillan, Chile, for pathological and histopathological study. Macroscopically, lungs appeared to be congested, edematous and "non-collapsed", which could be related to lesions compatible with interstitial pneumonia. Microscopic lesions showed a variable thickening of the septa, epithelial necrosis of the airways, diffuse inflammatory infiltration surrounding bronchial and bronchiolar areas, inflammatory exudate in the alveolar spaces with activate alveolar macrophages and fibrin. All lesions were compatible with Swine Influenza Virus (SIV) infection, and we successfully confirmed through SIV nucleoprotein immuno histochemistry staining of said samples, by utilizing a rapid and specific first-line diagnostic method that allows a rapid decision-making tool to control the spread of the virus. Additionally, the presence of the virus was confirmed by a serological field study.
\end{abstract}

Volume 5 Issue 6 - 2017

\author{
Tomás Casanova,' Daniela Rojas, ${ }^{2}$ Carolina \\ Baeza, ${ }^{3}$ Alvaro Ruiz ${ }^{2}$ \\ 'Depto Ciencias Clinicas, Universidad de Concepcion, Chile \\ ${ }^{2}$ Depto Patologia y Medicina Preventiva, Universidad de \\ Concepcion, Chile \\ ${ }^{3}$ Programa de Doctorado en Cs Veterinarias, Universidad de \\ Concepcion, Chile
}

\begin{abstract}
Correspondence: Alvaro Ruiz, Depto. Patologia y Medicina Preventiva, Facultad de Cs Veterinarias, Universidad de Concepcion, Chile.Avda.Vicente Méndez 595, Chillan, Chile, Tel +5642220 8787, Fax +5642227 320I, Email aruiz@udec.cl

Received: December 30, 2016 | Published: September 08, 2017
\end{abstract}

Keywords: Immunohistochemistry, diagnostic, antigen-detection, swine influenza virus, pigs

\section{Introduction}

Influenza A viruses are important in veterinary medicine because they cause influenza in horses, swine and birds, among other species. ${ }^{1}$ In humans, horses and swine, influenza is an acute respiratory disease that can become a major epidemic problem ${ }^{2-5}$ and cause local problems in production systems. ${ }^{6}$ Swine flu is an infectious disease caused by an influenza virus type A, characterized by the sudden onset of cough, dyspnea and fever, and is considered one of the most devastating swine respiratory diseases in intensive production systems. ${ }^{7}$ This infection has a significantly negative impact in the productive performance of animals, reducing average daily gains, increasing feed conversion efficiency and causing several other complications related to secondary infections by common agents in the respiratory tract. $^{8}$ Additionally, fever in animals could lead to miscarriages and reproductive failure. ${ }^{9}$ According to Donovan ${ }^{10} \&$ Dykhuis et al., ${ }^{11}$ the estimated cost of the infection in marked pigs ranges from 3.23 to 10.31 dollars per head. The use of a rapid and reliable diagnostic tool, which allows the producer to make the right decision, is essential. The aim of this study was to describe a rapid and specific immuno histochemistry (IHC) method in order to diagnose Swine influenza Virus Infection from field cases in diagnostic laboratories.

\section{Material and methods}

In order to confirm and evaluate the diagnosis of Swine Influenza Virus, the immunochemistry technique was performed on lung samples. The specimens, a total of three cuts per affected pig, with a positive control (donated by Dr. Carlos Perfumo, Universidad de La Plata, Argentina), were fixed in $4 \%$ buffered formalin solution, embedded in paraffin, and sectioned at $4 \mu \mathrm{m}$. The paraffin wax sections were rehydrated and its endogenous peroxidase activity was inhibited by $3 \% \mathrm{H}_{2} \mathrm{O}_{2}$ in methanol for 30 minutes and washed with phosphatebuffered saline solution (PBS) for 5 minutes. After hydration, sections of tissue were incubated in trypsin solution $(0.1 \%)$ in presence of calcium chloride dihydrate $3 \mathrm{M}$ for 20 minutes at $37^{\circ} \mathrm{C}$. Later on, slides were washed in PBS several times, and the samples were incubated overnight at $4^{\circ} \mathrm{C}$ with primary Anti-Influenza A Antibody, nucleoprotein, clones A1, A3 Blend (Millipore, MAB 8251) diluted 1:500 and the Cell Marque Ancillary Reagents Diamond: antibody diluent (Sigma-Aldrich, 938B). Negative control slides in absence of primary antibody but in presence of mouse $\mathrm{IgG}$ were included for each staining. ImmPRESSTM HRP Universal Antibody (Anti-Mouse IgG/Anti-Rabbit IgG, Peroxidase) Polymer Detection Kit (Vector Laboratories, MP-7500) was incubated at room temperature for 30minutes. Finally, ImmPACT DAB Peroxidase (HRP) Substrate kit (Vector Laboratories, SK-4105) was used to produce the color at the target site antigen to be recognized by the primary antibody. The nuclei were stained blue by the hematoxylin counterstain.

\section{Results}

Lung lesions were characterized by severe congestion, edema and interstitial pneumonia. As expected, histopathological analyses showed the same morphological patterns that were observed grossly, characterized by congestive interstitial blood vessels (pulmonary veins) and diffuse inflammatory infiltrate in the interstitial tissue. Additionally, severe bronchial and bronchiolar epithelial necrosis with cellular desquamation was observed (Figure 1A). The inflammatory infiltration was a mix of neutrophils and alveolar macrophages, with fibrin in the alveolar spaces forming hyaline membranes between cells (Figure 1B). Immunohistochemical staining of viral nucleoprotein showed intense viral replication in the lungs of pigs, located mainly in the airways, in the bronchial and bronchiolar epithelial cells (Figure 1C). In addition, specific cytoplasmic staining of alveolar macrophages revealed strong uptake of viral particles, either by direct phagocytosis of the virus or by phagocytosis of influenza virusinfected cells (Figure 1D). 


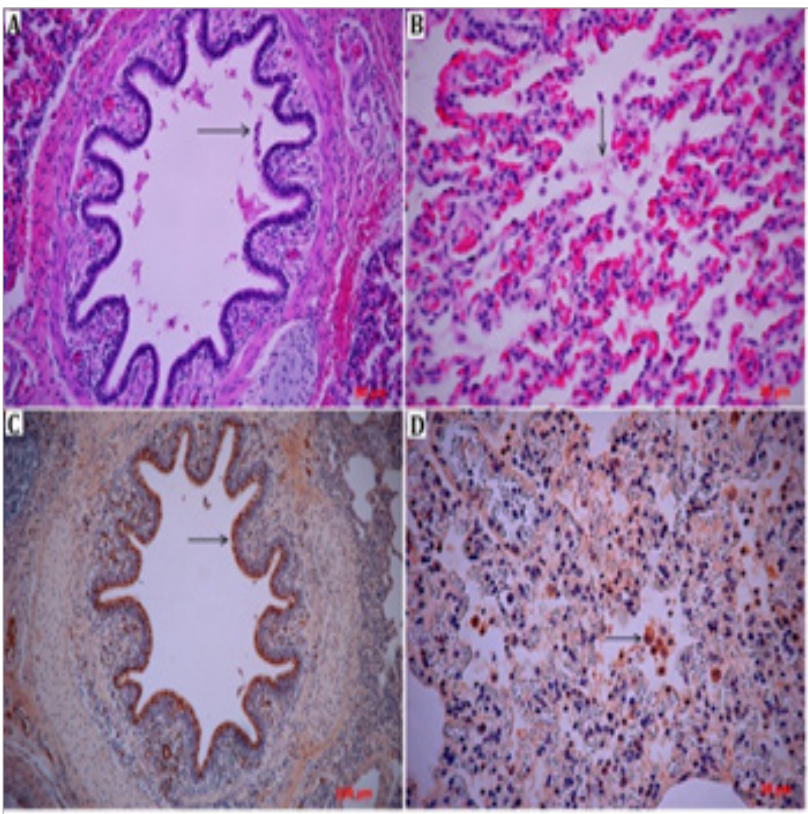

Figure I Lung histopathology (H\&E) of diseased pigs presents different levels of leukocyte inflammatory infiltration consisting predominantly of neutrophils, together with macrophages. In Section A, it is possible to identify severe bronchial epithelial cellular desquamation $(\rightarrow)$. Section B shows alveolar spaces containing numerous neutrophils and macrophages surrounded by fibrinous exudate $(\rightarrow)$. The expression and localization of the influenza virus antigen in lung tissue was detected by immunohistochemistry (sections $\mathrm{C}$ and D). Section $C$ shows the positive antigen detection in the ciliated epithelia of the bronchus and the macrophages in the lamina propria $(\rightarrow)$. Section D shows alveolar macrophages with positive staining with the anti-nucleoprotein antibody $(\rightarrow)$.

\section{Discussion}

Swine Influenza Virus is one of the most devastating respiratory diseases from the point of view of the health of the herd and in terms of productive parameters in pig farms. Cough, dyspnea, fever and anorexia are the more characteristic symptoms of the disease., ${ }^{2,7-11}$ In animals, the Influenza A virus infects the respiratory epithelium, causing necrosis and desquamation of the cells, therefore favoring its subsequent dissemination into the lower respiratory tract. In the alveolar space, the virus is picked up by alveolar macrophages and, together with intense phagocytic activity, it generates a swift inflammatory response. ${ }^{2,12}$ Through immunohistochemical staining, experimental infections in mice and pigs have shown that viral antigens are mainly detected in the epithelium of the airways and in alveolar macrophages, which have presented systematically higher immunoreactivity. ${ }^{12-14}$ Through IHC, we detected positivity towards infection by SIV in a natural presentation of a field virus, by using this technique as a rapid tool with in situ precision and reduced costs for the detection of this disease. Additionally, Capuccio ${ }^{15}$ evidenced differences between the intensity and distribution of the viral antigen in the lungs depending on which SIV strain was present and on the stage of infection, suggesting a new approach for greater precision in the etiological diagnosis of SIV that would complement molecular techniques with IHC. Compared to molecular techniques, the processing and sampling processes of IHC have a degree of rusticity, which can be considered a comparative advantage in field work. The development of a first-line diagnostic technique becomes an urgent need in order to allow the generation of quick responses to control the spread of Influenza Virus infection in pig farms. Immunohistochemistry is a technique that has a suitable specificity and sensitivity for highly accurate diagnosis of the Influenza Virus infection and enables identification of the virus as the direct cause of lesions in infected tissues and cells. ${ }^{16}$ For the Porcine Diagnostic Laboratory, the implementation of this technique has been a strategic tool serving as a constant support service for pork producers in central and southern Chile.

\section{Conclusion}

In conclusion, the development of the immunochemistry technique for the diagnosis of SIV in pigs, with compatible lesions of Influenza Virus by histopathological studies, serves as a real and accurate tool for its diagnosis in samples routinely submitted to the diagnostic laboratory.

\section{Acknowledgements}

None.

\section{Conflict of interest}

The author declares no conflict of interest.

\section{References}

1. Webster RG, Bean WJ, Gorman OT, et al. Evolution and ecology of influenza A viruses. Microbiol Rev. 1992;56(1):152-179.

2. Ciacci-Zanella JR, Schaefer R, Gava D, et al. Influenza A virus infection in Brazilian swine herds following the introduction of pandemic 2009 H1N1. Vet Microbiol. 2015;180(1-2):118-122.

3. Crisci E, Mussa T, Fraile L, et al. Review: influenza virus in pigs. Mol Immunol. 2013;55(3-4):200-221.

4. Franquiz MJ, Saleeb PG, Shanholtz CB, et al. Clinical characteristics of critically ill patients with suspected influenza during the 2009-10 and 2013-14 outbreaks. J Crit Care. 2016;20(38):73-77.

5. Suzuki Y. Sialobiology of influenza: molecular mechanism of host range variation of influenza viruses. Biol Pharm Bull. 2005;28(3):399-408.

6. Alexander DJ. A review of avian influenza in different bird species. Vet Microbiol. 2000;74(1-2):3-13.

7. Forrest HL, Webster RG. Perspectives on influenza evolution and the role of research. Anim Health Res Rev. 2010;11(1):3-18.

8. Cheong Y, Oh C, Lee K, et al. A survey of porcine respiratory disease complex (PRDC) associated pathogens among commercial pig farms of Korea via oral fluid method. J Vet Sci. 2016.

9. Zimmerman JJ, Karriker LA, Ramirez A, et al. Diseases of Swine. 10th ed. West Sussex, UK: Blackwell Publishing; 2012. p. 557-571.

10. Donovan TS. Influenza isolate selection methodology for timely autogenous vaccine use. The American Association of Swine Vet Ann Meeting San Diego, California USA; 2008. p. 557-561.

11. Dykhuis HC, Painter T, Fangman T, et al. Assessing production parameters and economic impact of swine influenza, PRRS and Mycoplasma hyopneumoniae on finishing pigs in a large production system. Vet. Ann. Meeting Denver, Colorado USA: The American Association of Swine; 2012. p. 75-76.

12. Casanova T, Van de Paar E, Desmecht D, et al. Hyporeactivity of alveolar macrophages and higher respiratory cell permissivity characterize DBA/2J mice infected by influenza a virus. J Interferon Cytokine Res. 2015;35(10):808-820. 
13. Qi L, Kash JC, Dugan VG, et al. The ability of pandemic influenza virus hemagglutinins to induce lower respiratory pathology is associated with decreased surfactant protein D binding. Virol J. 2011;412(2):426-434.

14. Kim HM, Lee YW, Lee KJ, et al. Alveolar macrophages are indispensable for controlling influenza viruses in lungs of pigs. $J$ Virol. 2008;82(9):426.
15. Cappuccio J, Dibarbora M, Lozada I, et al. Two years of surveillance of influenza a virus infection in a swine herd. Results of virological, serological and pathological studies. Comp Immunol Microbiol Infect Dis. 2017;50:110-115.

16. Pantin-Jackwood MJ. Immunohistochemical staining of influenza virus in tissues. Methods Mol Biol. 2014;1161:51-58. 